\section{MECHANISM OF ACTION OF LIVIDOMYCIN A, A NEW AMINOGLYGOSIDIC ANTIBIOTIC}

\section{Nobuyoshi Machiyama}

\author{
Institute of Applied Microbiology, \\ University of Tokyo, Tokyo
}

(Received for publication July 19, 1971)

Lividomycin A, a new aminoglycoside ${ }^{1,2)}$, was observed to inhibit protein synthesis more than nucleic acid syntheses in growing cells of Escherichia coli.

In a cell-free system, $f 2$ phage RNAdirected protein synthesis was markedly inhibited by the antibiotic. A similar inhibition was observed with streptomycin (Fig. 1).

Polyphenylalanine synthesis directed by poly $U$ in $E$. coli extract was also significantly affected by lividomycin A (Fig. 2). Inhibition of poly U-directed polypeptide synthesis was lower when $1 \mathrm{M} \quad \mathrm{NH}_{4} \mathrm{Cl}$ washed ribosomes and S-100 were employed. The methods and materials followed those described previously. ${ }^{3)}$

The incorporation of leucine, isoleucine and serine (miscoding) into polypeptide in the presence of poly $U$ was increased by the addition of lividomycin $\mathrm{A}$. The in vitro miscoding activity seemed to correspond to that of kanamycin (Table 1).

The acetylphenylalanyl-puromycin reaction, and $T$ factor- and $G$ factor associated GTPase reactions were not significantly

Table 1. In vitro miscoding produced by lividomycin $A$ in poly $U$ system.

\begin{tabular}{c|c|c|c|c}
\hline \multirow{2}{*}{ Antibiotics } & \multicolumn{4}{|c}{ Relative incorporation of } \\
\cline { 2 - 5 } & $\begin{array}{c}\text { Phenyl- } \\
\text { alanine }\end{array}$ & Leucine & $\begin{array}{c}\text { Iso- } \\
\text { leucine }\end{array}$ & Serine \\
\hline- & 100 & 5 & 1 & 0 \\
$\begin{array}{c}\text { Lividomycin A } \\
0.15 \mu \mathrm{g} / \mathrm{m} 1\end{array}$ & 62 & 7 & 3 & 1 \\
$\begin{array}{c}\text { Kanamycin } \\
5 \mu \mathrm{g} / \mathrm{ml} 1\end{array}$ & 26 & 7 & 4 & 1 \\
\hline
\end{tabular}

The reaction mixture contained in $0.2 \mathrm{ml}: E$. coli S-30 $1.4 \mathrm{mg}$, poly U $3 \mu \mathrm{g}$, tRNA $100 \mu \mathrm{g},{ }^{14} \mathrm{C}$-amino acid $0.02 \mu \mathrm{Ci}$, ATP $1 \mathrm{~mm}$, PEP $5 \mathrm{~mm}$, pyruvate kinase $4 \mu \mathrm{g}$, GTP $0.03 \mathrm{~mm}$, Tris- $\mathrm{HCl}, \mathrm{pH} 7.6,50 \mathrm{~mm}, \mathrm{NH}_{4} \mathrm{Cl}$ $100 \mathrm{~mm}, \mathrm{MgCl}_{2} 20 \mathrm{~mm}$, and 2-mercaptoethanol $6 \mathrm{~mm}$. It was incubated at $37^{\circ} \mathrm{C}$ for 40 minutes. $100=30.2$ pmoles. affected by lividomycin $\mathrm{A}$.

The results indicate that the primary site of action of lividomycin $A$ is in the bacterial system of protein synthesis. The mechanism of action of lividomycin A seems to be similar to that of other aminoglycosides such as streptomycin, kanamycin, paromomycin, etc.

Fig. 1. Effects of antibiotics on $\mathrm{f} 2 \mathrm{RNA}-$ directed protein synthesis.

The reaction mixture contained in $1.0 \mathrm{ml}$ : E. coli Q13 ribosomes (washed with $1 \mathrm{M} \mathrm{NH}{ }_{4} \mathrm{Cl}$ ) $58.2 \mathrm{~A}_{260}, \mathrm{~S}-1502 \mathrm{mg}$, initiation factors $345 \mu \mathrm{g}$, f 2 RNA $400 \mu \mathrm{g}, 19$ amino acids except valine $0.025 \mathrm{~mm}$, tRNA $200 \mu \mathrm{g}$, fMet-tRNA $150 \mu \mathrm{g},{ }^{14} \mathrm{C}-$ valine ( $169 \mathrm{mCi} / \mathrm{mmole}) 0.2 \mu \mathrm{Ci}, \mathrm{pH} 7.8$, Tris$\mathrm{HCl} 50 \mathrm{~mm}, \mathrm{NH}_{4} \mathrm{Cl} 100 \mathrm{~mm}, \mathrm{Mg}(\mathrm{AcO})_{2} 8 \mathrm{mM}$ DTT $1 \mathrm{~mm}$, ATP $1 \mathrm{~mm}$, PEP $5 \mathrm{~mm}$, pyruvate kinase $4 \mu \mathrm{g}$, and GTP $0.03 \mathrm{~mm}$.

pmoles/tube

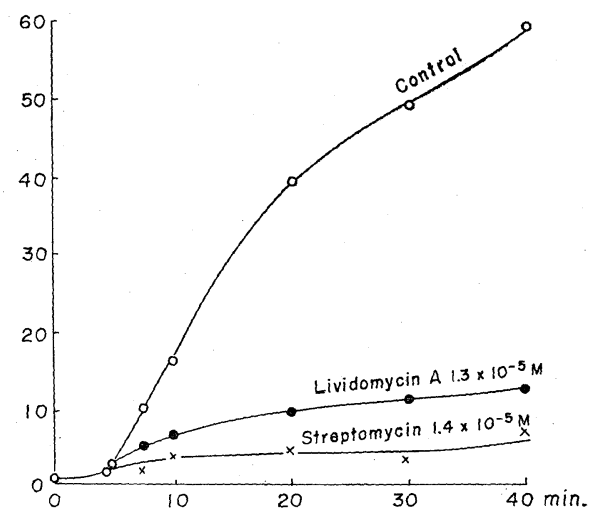

Fig. 2. Effects of antibiotics on polyphenylalanine synthesis.

The reaction mixture contained in $0.2 \mathrm{ml}$. E. coli $\mathrm{S}-30870 \mu \mathrm{g}$ protein, poly U $10 \mu \mathrm{g}$, tRNA $10 \mu \mathrm{g},{ }^{14} \mathrm{C}$-phenylalanine $(405 \mathrm{mCi} / \mathrm{mmole}) 0.04$ $\mu \mathrm{Ci}$, ATP $1 \mathrm{mM}$, PEP $5 \mathrm{~mm}$, pyruvate kinase $4 \mu \mathrm{g}$, GTP $0.03 \mathrm{mM}, \mathrm{pH} 7.8$, Tris-HCl $50 \mathrm{~mm}$, $\mathrm{NH}_{4} \mathrm{Cl} 100 \mathrm{mM}, \mathrm{MgCl}_{2} 10 \mathrm{~mm}$, and 2-mercaptoethanol $6 \mathrm{~mm}$.

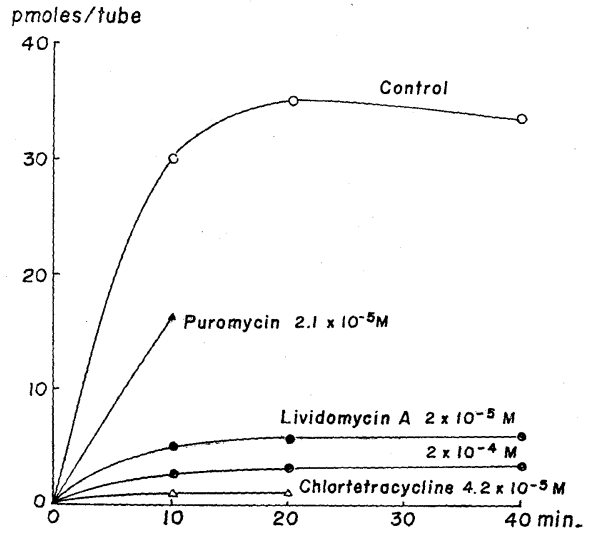


The author deeply appreciates the kind directions of Prof. $\mathrm{H}$. Umezawa and $\mathrm{N}$. TANAKA during this study.

\section{References}

1) Mori, T.; T. Ichiyanagi, H. Kondo, K. Tokunaga, T. Oda \& K. Munakata: Studies on new antibiotic lividomycins. II. Isolation and characterization of lividomycins A, B and other aminoglycosidic antibiotics pro- duced by Streptomyces lividus. J. Antibiotics $24: 339 \sim 346,1971$

2) Oda, T.; T. Mori, Y. Kyotani \& M. NakaYAMA: Studies on new antibiotic, lividomycins. IV. Structure of lividomycin A. J. Antibiotics $24: 511 \sim 518,1971$

3) Okuyama, A.; N. Machiyama, T. Kinoshita \& N. TANAKA: Inhibition by kasugamycin of initiation complex formation on $30 \mathrm{~S}$ ribosomes. Biochem. Biophys. Res. Communs. 43 : 196 199, 1971 